\title{
THE DARK SIDE OF ROTSE-III PROMPT GRB OBSERVATIONS
}

\author{
S. A. Yost, ${ }^{1}$ F. Aharonian, ${ }^{2}$ C. W. Akerlof, ${ }^{1}$ M. C. B. Ashley, ${ }^{3}$ S. Barthelmy, ${ }^{4}$ N. Gehrels, ${ }^{4}$ E. GöĞÜș, ${ }^{5}$ T. Güver, ${ }^{6}$ \\ D. Horns, ${ }^{2}$ Ü. Kizıloğlu, ${ }^{7}$ H. A. Krimm, ${ }^{4,8}$ T. A. McKay, ${ }^{1}$ M. Özel, ${ }^{9}$ A. Phillips, ${ }^{3}$ R. M. Quimby, ${ }^{10}$ \\ G. Rowell, ${ }^{2,11}$ W. Rujopakarn, ${ }^{12}$ E. S. Rykoff, ${ }^{1}$ B. E. Schaefer, ${ }^{13}$ D. A. Smith, ${ }^{1,14}$ H. F. Swan, ${ }^{1}$ \\ W. T. Vestrand, ${ }^{15}$ J. C. WheEler, ${ }^{10}$ J. Wren, $^{15}$ and F. Yuan ${ }^{1}$ \\ Received 2007 May 4; accepted 2007 July 16
}

\begin{abstract}
We present several cases of optical observations during $\gamma$-ray bursts (GRBs) which resulted in prompt limits but no detection of optical emission. These limits constrain the prompt optical flux densities and the optical brightness relative to the $\gamma$-ray emission. The derived constraints fall within the range of properties observed in GRBs with prompt optical detections, although at the faint end of optical $/ \gamma$-ray flux ratios. The currently accessible prompt optical limits do not require a different set of intrinsic or environmental GRB properties, relative to the events with prompt optical detections.
\end{abstract}

Subject headings: gamma rays: bursts

Online material: color figure

\section{INTRODUCTION}

Since the launch of the Swift satellite (Gehrels et al. 2004), early long-wavelength observations of $\gamma$-ray bursts (GRBs) have become routine. Swift has provided prompt triggers to events since early 2005, for which "prompt" signifies "during $\gamma$-ray emission." There is a growing number of optical light curves that begin during, or within seconds after, the $\gamma$-ray emission. There are also several cases with prompt optical nondetections which constrain the optical brightness during the GRB.

Prompt and very early broadband emission has been the major advance in Swift-era GRB studies, opening up serious investigations of important physical questions. One example is the nature

1 University of Michigan, 2477 Randall Laboratory, 450 Church St., Ann Arbor, MI, 48104; sayost@umich.edu, akerlof@umich.edu, tamckay@umich.edu, erykoff@umich.edu,donaldas@umich.edu,hswan@umich.edu, yuanfang@umich .edu.

2 Max-Planck-Institut für Kernphysik, Saupfercheckweg 1, 69117 Heidelberg, Germany; Felix.Aharonian@mpi-hd.mpg.de, horns@mpi-hd.mpg.de, rowell@ mpi-hd.mpg.de.

3 School of Physics, Department of Astrophysics and Optics, University of New South Wales, Sydney, NSW 2052, Australia; mcba@phys.unsw.edu.au, a.phillips@unsw.edu.au.

4 NASA/Goddard Space Flight Center, Greenbelt, MD 20771; scott@lheamail .gsfc.nasa.gov, gehrels@gsfc.nasa.gov, krimm@milkyway.gsfc.nasa.gov.

5 Sabancı University, Orhanl1-Tuzla 34956, Istanbul, Turkey; ersing@sabanciuniv .edu.

6 Istanbul University Science Faculty, Department of Astronomy and Space Sciences, 34119, University-Istanbul, Turkey; tolga@istanbul.edu.tr.

7 Middle East Technical University, 06531 Ankara, Turkey; umk@astroa physics.metu.edu.tr.

8 Universities Space Research Association, 10211 Wincopin Circle, Suite 500, Columbia, MD 21044-3432; krimm@milkyway.gsfc.nasa.gov.

9 Çanakkale Onsekiz Mart Üniversitesi, Terzioğlu 17020, Çanakkale, Turkey; m.e.ozel@comu.edu.tr.

${ }^{10}$ Department of Astronomy, University of Texas, Austin, TX 78712; quimby@ astro.as.utexas.edu,wheel@astro.as.utexas.edu.

11 School of Chemistry and Physics, University of Adelaide, Adelaide 5005, Australia;rowell@mpi-hd.mpg.de.

12 Steward Observatory Tucson, AZ 85721; wiphu@as.arizona.edu.

13 Department of Physics and Astronomy, Louisiana State University, Baton Rouge, LA 70803; schaefer@1su.edu.

14 Guilford College, 5800 West Friendly Avenue, Greensboro, NC 27410; dsmith4@guilford.edu.

15 Los Alamos National Laboratory, NIS-2 MS D436, Los Alamos, NM 87545; vestrand@lanl.gov, jwren@nis.lanl.gov. of the relativistic outflow, generally thought of as baryonic with energy released by internal shocks. The proposed alternatives include magnetized flows which release energy via magnetic reconnection (Meszaros et al. 1994; Thompson 1994; Usov 1994). The early broadband detections at X-ray and optical wavelengths are now being used to test these models (e.g., Kumar et al. 2007).

From the beginning of the afterglow discovery era, optical counterparts have been found to have a large range in brightness. Despite good observations, a significant fraction $(\sim 50 \%)$ of events do not have detected optical afterglows. These "optically dark" GRBs have generated questions regarding GRB physics and environment (see pre- and post-Swift reviews, such as Piran 2005 and Zhang 2007, respectively).

Nondetections during prompt optical observations are not precisely the same as these optically dark GRBs. In a few events, deeper post-GRB observations detect the optical transient. This raises the question as to whether prompt limits are "promptly dark"; are the limiting fluxes consistent with the brightness range observed in prompt optical detections, or do prompt nondetections require a separate population of optical properties? Such properties could be due to either intrinsic (faint events, or faint optical-to- $\gamma$-ray flux ratios) or extrinsic (local dust absorption, or the Ly $\alpha$ forest absorption from high $z$ ) causes.

"Excessively" faint prompt optical emission would therefore have interesting implications for the GRB spectral shape or environment. While the peak frequency of the GRB has often been constrained ( $\nu f_{\nu}$ peaking near a few $100 \mathrm{keV}$; see the review by Piran 2005), the shape of the prompt emission's low-energy tail is not well known, with self-absorption frequency estimates from the optical to X-ray (e.g., Pe'er \& Waxman 2004; Wei 2007). Indications of high redshift would also be important. While there are suggested redshift indicators from GRB $\gamma$-ray properties alone, these have not been proven, as discussed critically by Butler et al. (2007).

The ROTSE-III project has provided some of the earliest optical observations of GRB triggers, with a number of detections. To date, there has been no consistent correlation between prompt optical fluxes and the contemporaneous $\gamma$-rays (see, e.g., the discussions in Rykoff et al. 2005 and Yost et al. 2007). This paper discusses prompt ROTSE-III observations under good sky conditions which did not yield detections. The limits placed on the 

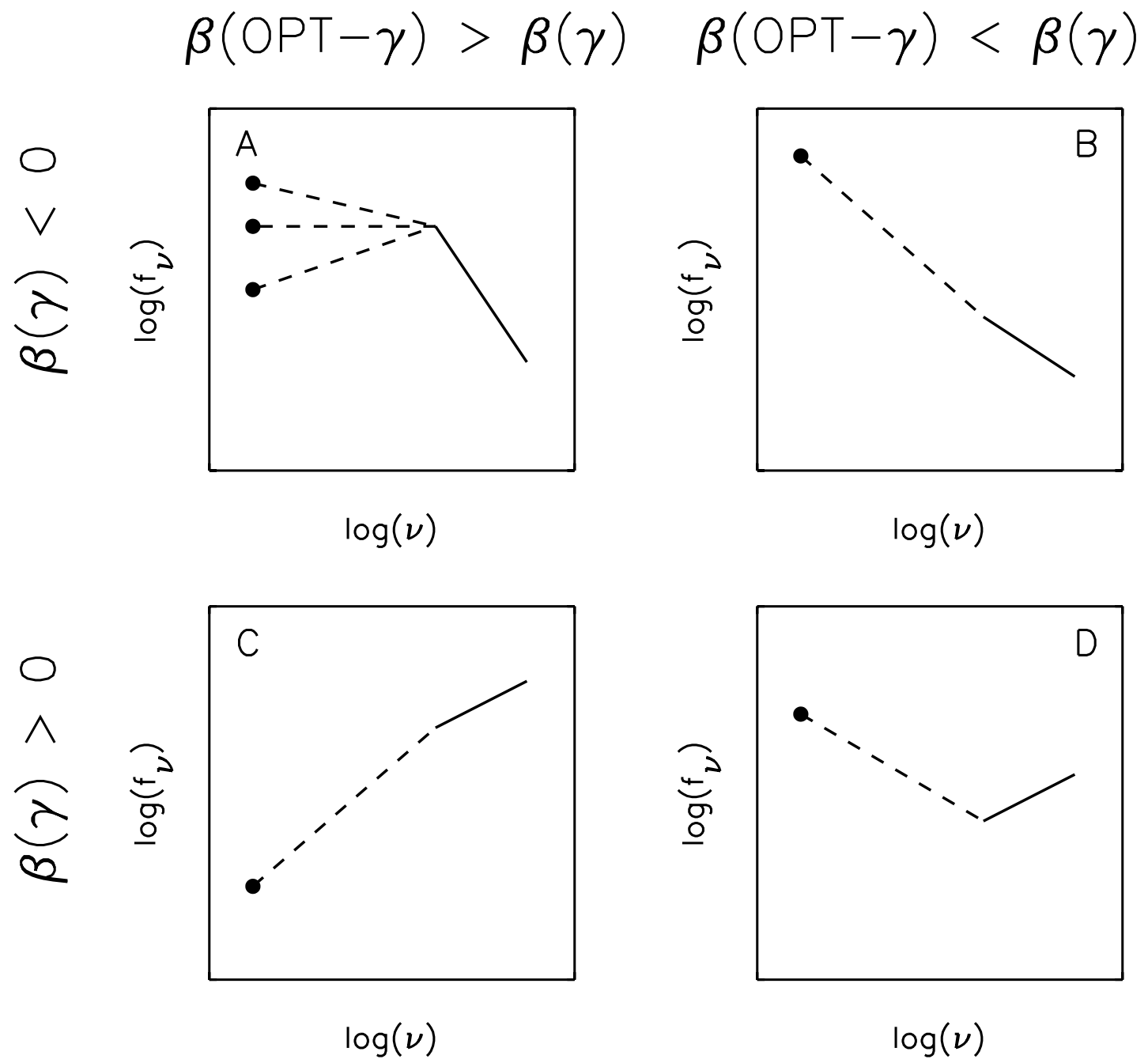

FIG. 1.-Diagrams representing some possible optical-to- $\gamma$-ray spectra. These illustrate information available from the spectral indices $\beta_{\gamma}$ (solid lines) and $\beta_{\text {opt- } \gamma}$ (dashed lines). In nearly all observed GRBs, the spectrum at the lower energy $\gamma$-rays (BAT band) has $\beta_{\gamma}<0$, as in the two upper panels. Rarely, $\beta_{\gamma}>0$ (lower panels). The comparison of the two $\beta$ constrains whether the $\gamma$-ray spectrum overpredicts (left, with $\beta_{\mathrm{opt}-\gamma}>\beta_{\gamma}$ ) or underpredicts (right, with $\beta_{\mathrm{opt}-\gamma}<\beta_{\gamma}$ ) the optical flux. With optical limits, an underprediction by $\beta_{\gamma}$ (optical excess) cannot be inferred, but an overprediction can be deduced; spectra allowing (B) or (A) can be differentiated from cases which only allow (A), and those congruent with (D) or (C) from those which only permit (C).

ratio of optical emission to the higher energy emission are discussed in comparison with the behavior associated with prompt detections.

In the following discussion, the spectral flux density is characterized by the spectral index $\beta$, with $f_{\nu} \propto \nu^{\beta}$. This convention relates $\beta$ to the $\gamma$-ray photon index $\Gamma$ by $\beta=1-\Gamma$. To designate a spectral region, subscripts "opt", "X", and " $\gamma$ " for $\beta$ indicate an index for the optical, X-ray, and $\gamma$-ray bands, respectively. A spectral index spanning two regions is designated with both, e.g., $\beta_{\text {opt }-\gamma}$ for the spectral index interpolating between the optical and $\gamma$-ray frequencies.

We note briefly that the overall spectral and temporal shape of afterglows typically suggests synchrotron emission from a fireball whose accelerated electrons have a Lorentz factor distribution $N\left(\gamma_{e}\right) \propto \gamma_{e}^{-p}$ (this is reviewed, e.g., by Meszaros 2006). The afterglow spectrum has spectral breaks, principally $\nu_{m}$, due to the minimum Lorentz factor $\gamma_{e}$, and $\nu_{c}$, the cooling frequency. These provide predictions for the spectral shape of a single synchrotron component. The index $\beta=1 / 3$ at frequencies below the peak in $f_{\nu}\left(\nu<\nu_{m}\right), \beta=(1-p) / 2$ for $\nu_{m}<\nu<\nu_{c}$, and $\beta=-p / 2$ for the case when $\nu>\nu_{c}$ and $\nu>\nu_{m}$. (When $\nu_{c}<\nu_{m}$, the spectral shape is $\nu^{-1 / 2}$ for frequencies between them.) These predictions, with $\beta$ from $1 / 3$ to $-3 / 2$ for $p=2-3$, can be compared to the constraints on $\beta_{\text {opt }-\gamma}$.

Figure 1 shows some possible combinations of $\beta_{\mathrm{opt}-\gamma}$ and $\beta_{\gamma}$. The $\gamma$-ray spectrum may predict the optical flux $\left(\beta_{\mathrm{opt}-\gamma}=\beta_{\gamma}\right)$, indicating that a single power-law (synchrotron-like) component could account for the broadband spectrum. When $\beta_{\text {opt }-\gamma}<\beta_{\gamma}$, the $\gamma$-ray spectrum underpredicts the optical flux, implying a separate low-energy emission component. When $\beta_{\text {opt }-\gamma}>\beta_{\gamma}$, the $\gamma$-ray spectrum overpredicts the optical flux, indicating a spectral rollover between the optical and high frequencies. When there are only prompt optical upper limits in flux density, one can nevertheless discriminate between cases where $\beta_{\gamma}$ either predicts or overpredicts the optical flux limit from those where $\beta_{\gamma}$ could underpredict the optical flux.

\section{OPTICAL OBSERVATIONS}

The ROTSE-III array is a worldwide network of $0.45 \mathrm{~m}$ robotic, automated telescopes, built for fast $(\sim 6 \mathrm{~s})$ responses to GRB triggers from satellites such as Swift. They have a wide $(1.85 \times 1.85)$ field of view imaged onto a Marconi $2048 \times 2048$ back-illuminated thinned CCD, and operate without filters. The ROTSE-III systems are described in detail in Akerlof et al. (2003). 
TABLE 1

Prompt Optical Limits

\begin{tabular}{|c|c|c|c|}
\hline GRB & $\begin{array}{c}t_{\text {start }} \\
\text { (s) }\end{array}$ & $\begin{array}{l}t_{\text {end }} \\
(\mathrm{s})\end{array}$ & Magnitude \\
\hline \multirow[t]{9}{*}{050306} & 64.8 & 69.8 & $>15.5$ \\
\hline & 78.9 & 83.9 & $>15.8$ \\
\hline & 93.5 & 98.5 & $>15.8$ \\
\hline & 108.3 & 113.3 & $>15.7$ \\
\hline & 122.7 & 127.7 & $>15.7$ \\
\hline & 137.2 & 142.2 & $>15.8$ \\
\hline & 151.8 & 156.8 & $>15.8$ \\
\hline & 166.1 & 171.1 & $>15.7$ \\
\hline & 180.4 & 185.4 & $>15.8$ \\
\hline \multirow[t]{2}{*}{ 050713A } & 72.1 & 77.1 & $>16.5$ \\
\hline & 104.7 & 124.7 & $>17.2$ \\
\hline \multirow[t]{6}{*}{$050822 \ldots \ldots \ldots$} & 31.8 & 36.8 & $>15.6$ \\
\hline & 39.8 & 44.8 & $>15.5$ \\
\hline & 47.8 & 52.8 & $>15.5$ \\
\hline & 55.9 & 60.9 & $>15.5$ \\
\hline & 63.9 & 68.9 & $>15.5$ \\
\hline & 95.9 & 100.9 & $>15.6$ \\
\hline 050915A & 42.9 & 47.9 & $>17.0$ \\
\hline \multirow[t]{2}{*}{ 050922B } & 258.6 & 263.6 & $>16.4$ \\
\hline & 273.3 & 278.3 & $>16.5$ \\
\hline \multirow[t]{8}{*}{051001} & 85.7 & 90.7 & $>16.3$ \\
\hline & 100.1 & 105.1 & $>16.3$ \\
\hline & 114.3 & 119.3 & $>16.2$ \\
\hline & 128.6 & 133.6 & $>16.3$ \\
\hline & 143.1 & 148.1 & $>16.3$ \\
\hline & 157.6 & 162.6 & $>16.2$ \\
\hline & 172.3 & 177.3 & $>16.1$ \\
\hline & 186.9 & 191.9 & $>16.2$ \\
\hline \multirow[t]{5}{*}{$060312 \ldots \ldots \ldots \ldots \ldots \ldots \ldots \ldots \ldots \ldots \ldots$} & 20.3 & 25.3 & $>14.1$ \\
\hline & 27.4 & 32.4 & $>14.1$ \\
\hline & 34.4 & 39.4 & $>14.2$ \\
\hline & 41.5 & 46.5 & $>14.3$ \\
\hline & 48.7 & 53.7 & $>14.3$ \\
\hline $060515 \ldots \ldots$ & 58.5 & 63.8 & $>14.5$ \\
\hline
\end{tabular}

ROTSE-III images were reduced and processed using the RPHOT pipeline, with routines based on DAOPHOT (Stetson 1987). Objects were identified via SExtractor (Bertin \& Arnouts 1996) and calibrated astrometrically and photometrically with the USNOB1.0 catalog. They are tied to the $R$ band, and these unfiltered " $R$-equivalent" magnitudes are designated as " $C_{R}$ ". The method is fully described in Quimby et al. (2006c). The final result yields limiting magnitudes in the GRB error box from the PSF-fit photometric data. These are presented in Table 1.

\subsection{Sources of Prompt Detection Data}

Table 2 presents spectral index information for several GRBs with prompt optical detections. These are used to provide a comparison for prompt limit results. The table is similar to Table 5 of Yost et al. (2007), which is also used for comparison.

Most of the prompt optical detections used for this table are from ROTSE-III observations. These include GRB 060111B (Yost et al. 2006), GRB 060729 (Quimby et al. 2006b), GRB 060904B (Rykoff et al. 2006), and GRB 061007 (Rykoff \& Rujopakarn 2006), which are discussed in a comprehensive analysis paper (E. S. Rykoff et al., in preparation). GRB 061121 was promptly detected by ROTSE; these data are presented in Page et al. (2007). GRB 060927 was a high-redshift event (Fynbo et al. 2006). The prompt ROTSE detection is converted to a flux density at a wavelength near $i$-band, as described in Ruiz-Velasco et al. (2007).
TABLE 1 - Continued

\begin{tabular}{|c|c|c|c|}
\hline GRB & $\begin{array}{c}t_{\text {start }} \\
(\mathrm{s})\end{array}$ & $\begin{array}{l}t_{\text {end }} \\
(\mathrm{s})\end{array}$ & Magnitude \\
\hline \multirow[t]{6}{*}{060614} & 26.8 & 31.8 & $>15.7$ \\
\hline & 40.6 & 45.6 & $>15.6$ \\
\hline & 55.2 & 60.2 & $>15.6$ \\
\hline & 69.6 & 74.6 & $>15.6$ \\
\hline & 83.9 & 88.9 & $>15.6$ \\
\hline & 98.3 & 103.3 & $>15.6$ \\
\hline a & 112.6 & 117.6 & $>15.6$ \\
\hline a & 126.8 & 131.8 & $>15.6$ \\
\hline a & 140.7 & 145.7 & $>15.6$ \\
\hline a & 155.2 & 160.2 & $>15.6$ \\
\hline a & 169.2 & 189.2 & $>16.2$ \\
\hline 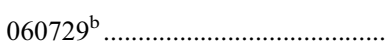 & 64.3 & 69.3 & $>16.6$ \\
\hline 061110 & 43.5 & 48.5 & $>16.4$ \\
\hline \multirow[t]{10}{*}{$061222 .}$. & 47.2 & 52.2 & $>17.0$ \\
\hline & 54.2 & 59.2 & $>16.9$ \\
\hline & 61.2 & 66.2 & $>17.0$ \\
\hline & 68.2 & 73.2 & $>16.9$ \\
\hline & 75.2 & 80.2 & $>16.9$ \\
\hline & 82.2 & 87.2 & $>16.9$ \\
\hline & 89.2 & 94.2 & $>17.0$ \\
\hline & 96.2 & 101.2 & $>16.9$ \\
\hline & 103.2 & 108.2 & $>16.9$ \\
\hline & 110.1 & 115.1 & $>17.0$ \\
\hline
\end{tabular}

Notes.-All times are in seconds since the burst onset, which are (UT): 03:33:12 UT (GRB 050306), 04:29:02.4 (GRB 050713A), 03:49:29 (GRB 050822), 11:22:42 (GRB 050915A), 15:02:00 (GRB 050922B), 11:11:36.2 (GRB 051001), 01:36:12.8 (GRB 060312), 02:27:52 (GRB 060515), $12: 43: 48.5$ (GRB 060614), 19:12:29.2 (GRB 060729), 11:47:21.3 (GRB 061110), and 03:28:52.1 (GRB 061222). Magnitudes are quoted without correction for local or Galactic extinction, and are $R$-equivalent unfiltered values. The extinction corrections are (in $A_{R}$ magnitudes): 1.817 (GRB 050306), 1.107 (GRB 050713A), 0.04 (GRB 050822), 0.07 (GRB 050915A), 0.098 (GRB 050922B), 0.04 (GRB 051001), 0.472 (GRB 060312), 0.073 (GRB 060515), 0.058 (GRB 060614), 0.145 (GRB 060729), 0.242 (GRB 061110), and 0.266 (GRB 061222).

a The Swift UVOT detected the OT in this event during an exposure from 102-202 s post-trigger (Parsons et al. 2006). The ROTSE limits are consistent with the more sensitive UVOT detection.

${ }^{\mathrm{b}}$ GRB 060729 was promptly detected; however, the first $5 \mathrm{~s}$ observation only yielded a limit for the OT.

GRB 060218 was detected by ROTSE (Table 2 , line with a $C_{R}$ observation of GRB 060218; Quimby et al. 2006a) and by the Swift UVOT (Table 2, line with a $V$ observation of GRB 060218 ; Campana et al. 2006). Finally, GRB 050820A and GRB 061126 were promptly detected by RAPTOR. For the former, we determine optical and $\gamma$-ray flux densities from Vestrand et al. (2006). For the latter, we take the optical flux densities of Perley et al. (2007), correcting for Galactic extinction.

\subsection{Prompt Nondetections with Later Detections}

One case of a prompt limit with a later detection is the first ROTSE observation of GRB 060729. The OT flux was rising, and the second $5 \mathrm{~s}$ image was the first to yield a detection (Tables 2 and 3 show that the flux rises from $\sim 1 / 2$ to 2 mJy over the first few images).

In GRB 060614, the ROTSE limits at 29 s post-trigger were obtained before the subsequent UVOT afterglow detection at 100 s post-trigger. This initial UVOT $V$-band detection (Parsons et al. 2006) had notable flux uncertainty $(18.4 \pm 0.5 \mathrm{mag})$, but is significantly (nearly $3 \mathrm{mag}$ ) fainter than the ROTSE limits. The ROTSE limit values are fully consistent with the later detection, 
TABLE 2

Spectral Indices $\beta_{\mathrm{opt}-\gamma}\left(\mathrm{or} \beta_{\mathrm{opt}-\mathrm{X}}\right)$ From Prompt Optical Detections

\begin{tabular}{|c|c|c|c|c|c|c|c|c|}
\hline GRB & $\begin{array}{l}t_{\text {start }} \\
(\mathrm{s})\end{array}$ & $\begin{array}{l}t_{\text {end }} \\
(\mathrm{s})\end{array}$ & Band & $\begin{array}{r}f_{\nu}(\mathrm{opt}) \\
(\mathrm{mJy})\end{array}$ & $\begin{array}{l}\nu_{\gamma}\left(\text { or } \nu_{\mathrm{X}}\right) \\
\left(\times 10^{18} \mathrm{~Hz}\right)\end{array}$ & $\begin{array}{c}f_{\nu}(\gamma) \underset{\left[\operatorname{or} f_{\nu}(\mathrm{X})\right]}{(\mu \mathrm{Jy})} \\
\end{array}$ & $\beta_{\gamma}\left(\right.$ or $\left.\beta_{\mathrm{X}}\right)$ & $\beta_{\mathrm{opt}-\gamma}\left(\right.$ or $\left.\beta_{\mathrm{opt}-\mathrm{X}}\right)$ \\
\hline 050820A........ & 252 & 282 & $C_{R}$ & $2.612 \pm 0.058$ & 25 & $453 \pm 17$ & $-0.371 \pm 0.061$ & $-0.161 \pm 0.004$ \\
\hline 050820A ..................... & 515 & 545 & $C_{R}$ & $4.452 \pm 0.077$ & 27 & $138 \pm 15$ & $-0.707 \pm 0.143$ & $-0.32 \pm 0.01$ \\
\hline 060111B ..................... & 58.0 & 63.0 & $C_{R}$ & $5.97 \pm 0.66$ & 14 & $89 \pm 14$ & $-1.02 \pm 0.20$ & $-0.41 \pm 0.02$ \\
\hline $060218 \ldots \ldots \ldots \ldots \ldots \ldots \ldots$ & 691 & 1027 & $C_{R}$ & $0.254 \pm 0.026$ & 11 & $91.3 \pm 7.4$ & $-1.5 \pm 0.1$ & $-0.10 \pm 0.01$ \\
\hline $060729 \ldots \ldots \ldots \ldots \ldots \ldots \ldots \ldots$ & 92.9 & 97.9 & $C_{R}$ & $1.90 \pm 0.18$ & 14 & $173 \pm 15$ & $-0.986 \pm 0.087$ & $-0.23 \pm 0.02$ \\
\hline $060729 \ldots \ldots \ldots \ldots \ldots \ldots \ldots$ & 114.8 & 119.8 & $C_{R}$ & $0.74 \pm 0.18$ & 15 & $35.4 \pm 6.9$ & $-0.896 \pm 0.065$ & $-0.29 \pm 0.04$ \\
\hline $060729 \ldots \ldots \ldots \ldots \ldots \ldots \ldots$ & 128.8 & 133.8 & $C_{R}$ & $0.61 \pm 0.18$ & 15 & $20.0 \pm 6.3$ & $-0.896 \pm 0.065$ & $-0.33 \pm 0.04$ \\
\hline $060729 \ldots \ldots \ldots \ldots \ldots \ldots \ldots$ & $\ldots$ & $\ldots$ & $\ldots$ & $\ldots$ & 0.67 & $1527 \pm 34$ & $-2.004 \pm 0.029$ & $0.13 \pm 0.04$ \\
\hline 060904В ..................... & 18.5 & 31.5 & $C_{R}$ & $0.278 \pm 0.055$ & 18 & $20.52 \pm 6.3$ & $-0.416 \pm 0.081$ & $-0.288 \pm 0.035$ \\
\hline 060904B & 146.4 & 166.4 & $C_{R}$ & $0.370 \pm 0.072$ & 13 & $36.2 \pm 7.5$ & $-1.30 \pm 0.18$ & $-0.270 \pm 0.046$ \\
\hline 060904B .................... & 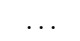 & $\ldots$ & $\ldots$ & $\ldots$ & 0.67 & $1011 \pm 29$ & $-1.26 \pm 0.03$ & $0.080 \pm 0.027$ \\
\hline 061007........................ & 77.8 & 82.8 & $C_{R}$ & $407.4 \pm 5.7$ & 16 & $215.9 \pm 12$ & $-0.673 \pm 0.072$ & $-0.72 \pm 0.01$ \\
\hline $061007 \ldots \ldots \ldots \ldots \ldots \ldots$ & 92.0 & 97.0 & $C_{R}$ & $500.5 \pm 7.8$ & 0.67 & $1400.4 \pm 8.2$ & $-0.906 \pm 0.013$ & $-0.810 \pm 0.002$ \\
\hline $061007 \ldots \ldots \ldots \ldots \ldots \ldots \ldots$ & & $\ldots$ & $\ldots$ & $\ldots$ & 15 & $92 \pm 12$ & $-0.824 \pm 0.093$ & $-0.828 \pm 0.013$ \\
\hline 061007....................... & 106 & 111 & $C_{R}$ & $449.0 \pm 6.4$ & 0.67 & $1118.0 \pm 6.0$ & $-0.906 \pm 0.013$ & $-0.826 \pm 0.002$ \\
\hline 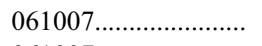 & $\ldots$ & $\ldots$ & $\ldots$ & $\ldots$ & 15 & $33.4 \pm 7.6$ & $-0.824 \pm 0.093$ & $-0.916 \pm 0.025$ \\
\hline 061007........................ & 120 & 125 & $C_{R}$ & $376.6 \pm 9.5$ & 0.67 & $909.9 \pm 4.5$ & $-0.906 \pm 0.013$ & $-0.830 \pm 0.004$ \\
\hline 061007........................ & $\ldots$ & $\ldots$ & $\ldots$ & $\ldots$ & 15 & $39.6 \pm 7.8$ & $-0.824 \pm 0.093$ & $-0.882 \pm 0.023$ \\
\hline $061007 \ldots \ldots \ldots \ldots \ldots \ldots \ldots$ & 135 & 140 & $C_{R}$ & $333.7 \pm 5.0$ & 0.67 & $760.5 \pm 3.5$ & $-0.906 \pm 0.013$ & $-0.838 \pm 0.002$ \\
\hline 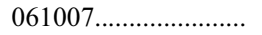 & & $\ldots$ & $\ldots$ & $\ldots$ & 15 & $26.0 \pm 7.2$ & $-0.824 \pm 0.093$ & $-0.911 \pm 0.030$ \\
\hline 061007....................... & 149 & 154 & $C_{R}$ & $280.9 \pm 4.9$ & 0.67 & $644.8 \pm 2.8$ & $-0.906 \pm 0.013$ & $-0.837 \pm 0.002$ \\
\hline $061007 \ldots \ldots \ldots \ldots \ldots \ldots$ & $\ldots$ & $\ldots$ & $\ldots$ & $\ldots$ & 15 & $24.9 \pm 7.1$ & $-0.824 \pm 0.093$ & $-0.899 \pm 0.031$ \\
\hline 061007......................... & 164 & 169 & $C_{R}$ & $233.5 \pm 4.7$ & 0.67 & $554.0 \pm 2.3$ & $-0.906 \pm 0.013$ & $-0.832 \pm 0.003$ \\
\hline 061007............................. & $\ldots$ & $\ldots$ & $\ldots$ & $\ldots$ & 15 & $21.6 \pm 2.6$ & $-0.824 \pm 0.093$ & $-0.895 \pm 0.014$ \\
\hline $061121 \ldots \ldots \ldots \ldots \ldots \ldots \ldots$ & $\ldots$ & $\ldots$ & $\ldots$ & $\ldots$ & 0.24 & $6930 \pm 320$ & $-0.07 \pm 0.08^{\mathrm{b}}$ & $0.118 \pm 0.046$ \\
\hline $061121 \ldots \ldots \ldots \ldots \ldots \ldots$ & 92.5 & 126 & $C_{R}$ & $1.04 \pm 0.51$ & 15 & $49.3 \pm 5.0$ & $-0.83 \pm 0.10$ & $-0.29 \pm 0.05$ \\
\hline $061121 \ldots \ldots \ldots \ldots \ldots \ldots \ldots$ & $\ldots$ & $\ldots$ & $\ldots$ & $\ldots$ & 0.24 & $2024 \pm 74$ & $-{ }^{\mathrm{c}}$ & $0.079 \pm 0.091$ \\
\hline $061126 \ldots \ldots \ldots \ldots \ldots \ldots$ & 20.9 & 25.9 & $C_{R}$ & $60.65 \pm 0.55$ & 19 & $473 \pm 16$ & $-0.262 \pm 0.059$ & $-0.459 \pm 0.007$ \\
\hline $061126 \ldots \ldots \ldots \ldots \ldots \ldots \ldots$ & 29.8 & 34.8 & $C_{R}$ & $41.96 \pm 0.77$ & 19 & $65.9 \pm 9.2$ & $\cdots$ & $-0.61 \pm 0.02$ \\
\hline $061126 \ldots \ldots \ldots \ldots \ldots \ldots \ldots$ & 38.6 & 43.6 & $C_{R}$ & $28.50 \pm 0.78$ & 19 & $47 \pm 12$ & $\ldots$ & $-0.60 \pm 0.03$ \\
\hline
\end{tabular}

Notes.-Optical and $\gamma$-ray flux densities and spectral indices correspond to the time intervals $t_{\text {start }}-t_{\text {end }}$ from the GRB trigger. The sources of the data are discussed in $\S 2.1$, and the optical data are corrected for Galactic extinction.

${ }^{a}$ Filterless observations of this high- $z$ event were calibrated to the flux density at $819 \mathrm{~nm}$, approximately $i$-band; see Ruiz-Velasco et al. (2007).

b Taken from the spread in spectral indices with different extinction models; see Page et al. (2007, Table 4).

c There is no value given by Page et al. (2007) for the spectral shape during the steep decline from the peak. For many cases, the steep X-ray phase has been reported as spectrally indistinguishable from the later shallow decay, but in some cases $\beta_{\mathrm{X}}$ is softer during the initial rapid decay (Nousek et al. 2006).

and constrain the flux decay to have been no more rapid than $\sim t^{-2}$ from 0.5 to 2 minutes post-trigger.

Optical detections indicate that the GRB cannot be at high $z$, as the Ly $\alpha$ forest would absorb the optical flux. Indeed, GRB 060729 has $z=0.54$ (Thoene et al. 2006), and the host of GRB 060614 is at $z=0.125$ (Price et al. 2006; although there is some controversy, with an estimate of $z \approx 1.5$; cf. Schaefer \& Xiao 2006). There are further GRBs with prompt limits followed by optical detections at $t \gtrsim 1 \mathrm{hr}$, such as GRB 050713A (Malesani et al. 2005) and GRB 061110 (Chen et al. 2006). The prompt nondetections of these events cannot be attributed to high $z$.

\section{HIGH-ENERGY DATA}

BAT data were used for $\gamma$-ray comparisons in these Swift bursts. For the $\gamma$-ray data, the event files from the public archives were analyzed with the BATTOOLS and XSPEC11 software packages. ${ }^{16}$

\footnotetext{
16 Available at: http://swift.gsfc.nasa.gov/docs/swift/analysis/.
} 
TABLE 3

Spectral Index $\beta_{\text {opt }-\gamma}$ Limits from Prompt Optical Limits

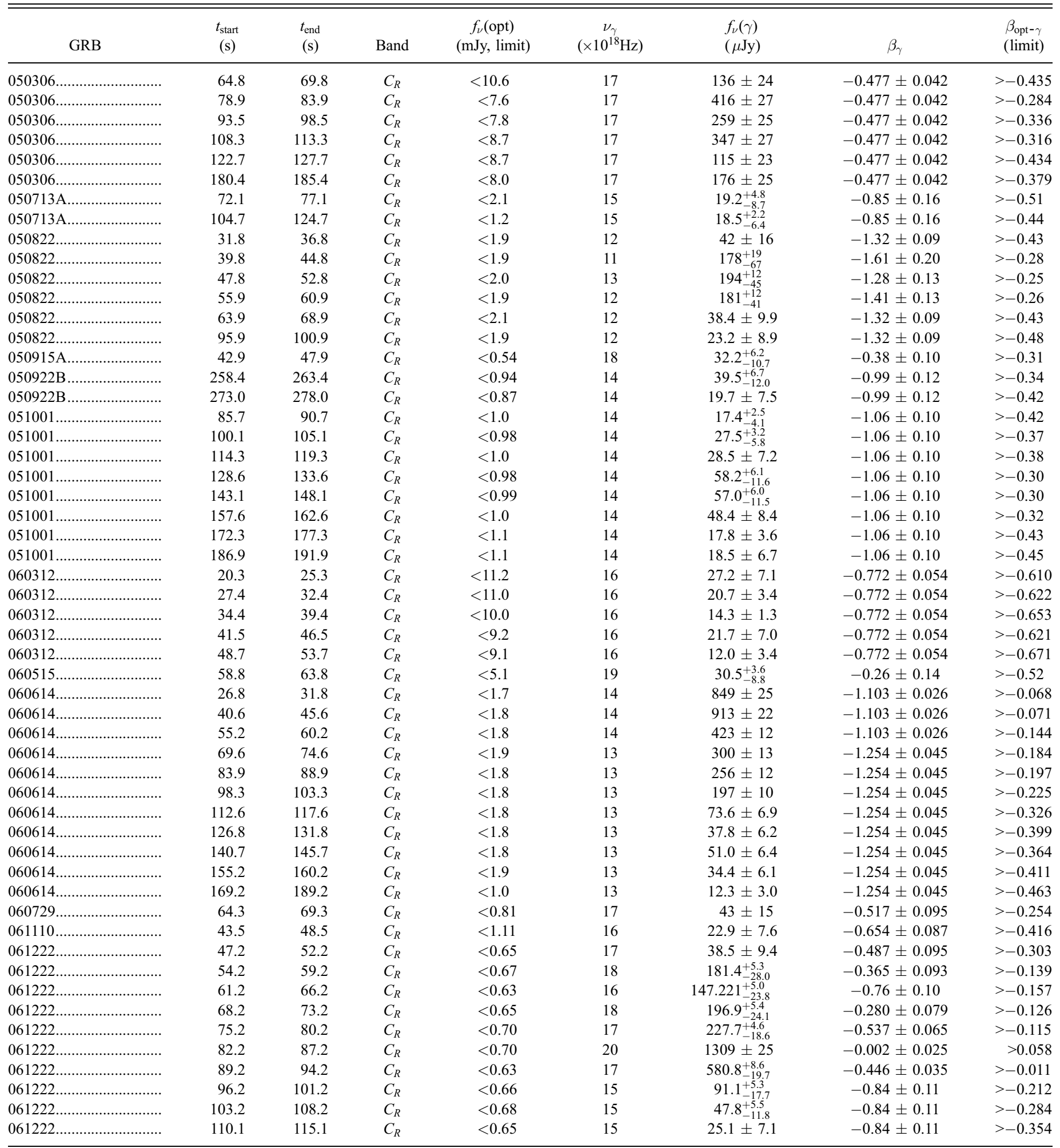

Notes.-Optical flux limits and $\gamma$-ray flux densities $f_{\nu}$ and their spectral indices, corresponding to the time intervals $t_{\text {start }}-t_{\text {end }}$ from the GRB trigger. The $\gamma$-ray count rates were all detected at the $3 \sigma$ level or better, although the spectral fits for some cases result in $f_{\nu}$ with signal-to-noise ratios formally $<3$. The optical limits are from Table 1, corrected for Galactic extinction. 
The result is unabsorbed flux values in the $15-150 \mathrm{keV}$ range. When there is sufficient signal (for $\approx 30 \%$ of the data points), these are determined directly along with $\beta_{\gamma}$ during the precise time interval of each optical observation. For the remainder of the data, where the signal is insufficient, the count rate during the interval is converted to fluxes using the BAT spectrum during a longer, overlapping interval. The analyses are the same as described for the GRB 051109A and GRB 051111 events in Yost et al. (2007).

In addition, a few events have prompt X-ray data (in the 1$10 \mathrm{keV}$ band, with an effective frequency $\nu<10^{18} \mathrm{~Hz}$ ) as well. Table 2 lists results with simultaneous optical, X-ray, and $\gamma$-ray detections for GRB 060729, GRB 060904B, and GRB 061007. The XRT analyses are fully discussed in an upcoming ROTSE paper treating multiband light curves (E. S. Rykoff et al., in preparation). In brief, the xrtpipeline tool calibrates and performs standard filtering and screening. This is followed by count extractions from appropriate regions for the source and background, the generation of response files with the FTOOLS task xrtmkarf, and spectral fits to yield fluxes. For GRB 061121, the XRT data are taken directly from the flux densities in Page et al. (2007); the reductions were similar and compensate for the significant pileup effects, as discussed there in detail.

There are two cases with optical nondetections and X-ray prompt detections within the sample presented, GRB 050713A and GRB 060614. A limit on $\beta_{\text {opt-X }}$ adds little to the information from the $\beta_{\text {opt }-\gamma}$ limit; the events are compatible with an interpretation of the prompt $t \approx 100 \mathrm{~s} \mathrm{X}$-ray flux as an extension of the contemporaneous $\gamma$-rays. This was seen in a quick analysis of the GRB 060614 archive data (as well as the spectral information given in Mangano et al. 2006 and Barthelmy et al. 2006), and by the O'Brien et al. (2006) analysis of GRB 050713A XRT and BAT data. Further detailed comparisons are beyond the scope of this paper.

\section{DETERMINING $\beta_{\text {opt- } \gamma}$ AND $\beta_{\text {opt-X }}$}

The spectral index (or its limit) was determined between the optical and higher energy bands in the same manner as those presented in Yost et al. (2007). In brief, the optical data were corrected for Galactic extinction and converted to flux densities as if the $C_{R}$ magnitudes were $R$, using the zero points of Bessell (1979). These data are in Tables 2 and 3, along with the flux densities of the $\gamma$-ray detections (and X-ray, where applicable). The flux densities and effective frequencies of the bands are then used to calculate $\beta$. When the optical is not detected, the optical limit is used with the lower $(1 \sigma)$ estimate of the high-energy emission to estimate the softest spectral index $\beta_{\text {opt- } \gamma}$ (or $\beta_{\text {opt-X}}$ ) possible.

The Galactic extinction corrections are taken from Schlegel et al. (1998). The $C_{R}$ limits are treated as $R$-equivalent and adjusted for the $R$ band's extinction. Table 2 gives the flux and $\beta$ results for cases with prompt optical detections, in the same manner as Yost et al. (2007). Table 3 gives flux and $\beta$ constraints for events with prompt optical limits.

\section{DISCUSSION}

We consider 27 GRBs with prompt optical observations, the data presented in Tables 2 and 3, as well as Table 5 in Yost et al. (2007). Of these GRBs, only 11 had prompt optical limits, while 14 were consistently promptly detected in the optical, and a further 2 events had both prompt limits and detections. The data include a total of 43 distinct prompt optical detections, and 55 prompt optical limits.

\subsection{Diverse Prompt Properties}

GRBs show diversity in their prompt optical and $\gamma$-ray brightnesses. Optical flux densities span $100 \mu \mathrm{Jy}$ to $3 \mathrm{Jy}$, while contem- poraneous $\gamma$-ray flux densities take values from $6 \mu \mathrm{Jy}$ to $4 \mathrm{mJy}$. This results in a range of possible prompt spectral indices $\beta_{\text {opt }-\gamma}$ and $\beta_{\gamma}$, which are plotted as $\beta_{\text {opt- } \gamma}$ versus $\beta_{\gamma}$ in Figure 2.

In this data set, all relations between $\beta_{\text {opt- } \gamma}$ and $\beta_{\gamma}$ are observed $\left(\beta_{\text {opt }-\gamma}>\beta_{\gamma}, \beta_{\text {opt }-\gamma}<\beta_{\gamma}\right.$, or $\left.\beta_{\text {opt- } \gamma}=\beta_{\gamma}\right)$. The values of $\beta_{\text {opt }-\gamma}$ and $\beta_{\gamma}$ vary widely, from -0.9 to 0.03 for $\beta_{\text {opt }-\gamma}$ and from -1.5 to 0.4 for $\beta_{\gamma}$. These are within or quite close to the range of $\beta=1 / 3$ to $-3 / 2$ for the synchrotron spectral shape (discussed in $\S 1$ ) and electron energy distribution indices of $p=2-3$.

In addition, observations of some events show both $\beta_{\text {opt }-\gamma}$ and $\beta_{\gamma}$ changing significantly during a burst. The value of $\beta_{\gamma}$ generally evolves from hard to soft. This is a previously known characteristic of many GRBs (e.g., as reviewed by Fishman \& Meegan 1995), now considered in models of prompt emission (such as "jitter" radiation; Medvedev 2006). The changes in $\beta_{\text {opt- } \gamma}$ indicate that optical prompt fluxes are generally not correlated with the $\gamma$-ray emission.

There has been discussion in the literature concerning whether prompt optical emission is an extension of the $\gamma$-rays, or is a separate component. Vestrand et al. (2005) indicate an optical component correlated to the GRB in GRB 041219A, while Vestrand et al. (2006) and Yost et al. (2007) discuss the apparent blend of $\gamma$-raycorrelated and uncorrelated components in the prompt optical light curves of GRBs 050820A and 051111, respectively. The correlated component of GRB 051111 is one of the few cases where the indices allow $\beta_{\text {opt }-\gamma}=\beta_{\gamma}$. Several events had prompt optical behavior distinct from that of the GRB, and apparently connected to the afterglow; the prompt optical light curves of GRB 050401, GRB 051109A, and GRB 061126 are decaying (Rykoff et al. 2005; Yost et al. 2007; Perley et al. 2007), and that of GRB 060729 is rising (Quimby \& Rykoff 2006). There are also events where the optical flux does not rise until after the GRB (e.g., GRB 030418, GRB 060605, GRB 060607A; Rykoff et al. 2004, Schaefer et al. 2006, Nysewander \& Haislip 2006, respectively).

As seen by the variety of $\beta_{\mathrm{opt}-\gamma}$, there is no universal ratio $f_{\nu}(\mathrm{opt}) / f_{\nu}(\gamma)$. There is no common $\beta_{\text {opt }-\gamma} / \beta_{\gamma}$ connection in all events, but in most cases, $\beta_{\text {opt }-\gamma}$ is harder than $\beta_{\gamma}$. For these, $\beta_{\gamma}$ overpredicts the optical, requiring a rollover in the spectrum between the $\gamma$-ray and optical frequencies, whether or not there are separate emission components at optical and $\gamma$-ray energies. Nearly all the limits give $\beta_{\mathrm{opt}-\gamma}$ versus $\beta_{\gamma}$ falling into this category.

In some prompt detections, $\beta_{\mathrm{opt}-\gamma}<\beta_{\gamma}$, and $\beta_{\gamma}$ underpredicts the optical (e.g., see Yost et al. 2007 and Perley et al. 2007 for GRB 051111 and GRB 061126, respectively). This implies a separate low-energy emission component. All the prompt limits presented exclude this possibility, except for GRB 060515 . Its constraints are insufficient and allow either $\beta_{\text {opt- } \gamma}<\beta_{\gamma}$ or $\beta_{\text {opt- } \gamma}>\beta_{\gamma}$.

\subsection{Properties of Limits vs. Detections}

The optical limits are not demonstrably the result of abnormally faint prompt optical flux. The prompt flux limits are typically 16 or $17 \mathrm{mag}(<1 \mathrm{mJy})$. Prompt detections have been recorded from small fractions of a mJy to a few Jy. In addition, the $\gamma$-ray flux densities of GRBs with prompt optical limits are similar to the lower values of $f_{\nu}(\gamma)$ from GRBs with optical detections; both sets of events have $f_{\nu}(\gamma)$ ranging from several $\mu \mathrm{Jy}$ to over $1 \mathrm{mJy}$. The prompt limits require neither intrinsically fainter emission nor excess absorption from dust or (high- $z$ ) Ly $\alpha$. High redshifts are not a general solution for the prompt optical limits, as some events are detected later $(\S 2.2)$.

The values of GRB $\beta_{\gamma}$ contemporaneous with prompt optical limits are similar to the $\beta_{\gamma}$ when prompt observations gave optical detections. The $\beta_{\gamma}$ of optical nondetections are on average softer than the $\beta_{\gamma}$ of detections, ranging from -1.6 to 0 , as compared to 


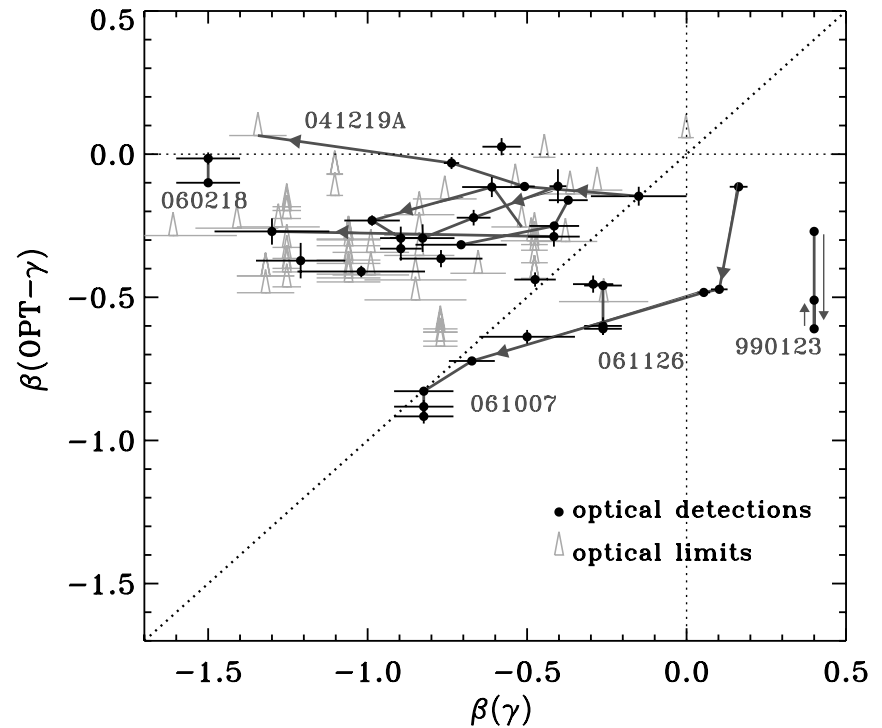

FIG. 2.-Optical-to- $\gamma$ spectral indices $\left(\beta_{\mathrm{opt}-\gamma}\right)$ plotted against $\gamma$-ray spectral indices $\left(\beta_{\gamma}\right)$. Data are from Tables 2 and 3, as well as Table 5 of Yost et al. (2007). Optical detections are shown by black filled circles, and cases with optical limits as gray triangles. The latter indicate the softest possible $\beta_{\text {opt- } \gamma}$; the triangles are used to point upwards instead of arrows. When several optical detections occur in a single GRB event, the points are connected by a line (colored in online version). Where legible, an arrow points from earlier to later observations. Most cases are above the $\beta_{\mathrm{opt}-\gamma}=\beta_{\gamma}$ line, with $\beta_{\mathrm{opt}-\gamma}>\beta_{\gamma}$. The $\gamma$-ray spectrum overpredicts the optical flux; this indicates a spectral rollover between the optical and high frequencies, whether or not there are separate emission components at optical and $\gamma$-ray energies. Sometimes $\beta_{\text {opt- } \gamma}<\beta_{\gamma}$. The $\gamma$-ray spectrum underpredicts the optical flux, implying a separate low-energy emission component. A few cases have consistent indices, which, as discussed for GRB 051111 (Yost et al. 2007), could indicate a single spectral shape extending from $\gamma$-ray to optical energies. The optical limits are consistent with $f_{\nu}(\mathrm{opt}) / f_{\nu}(\gamma)$ ratios from optical detections and do not imply a separate population whose prompt optical emission is fainter relative to the $\gamma$-rays. [See the electronic edition of the Journal for a color version of this figure .]

-1.5 to 0.4 . However, the data are not consistently sampled, leading to no strong conclusions other than that prompt observations of the few GRBs with the hardest $\beta_{\gamma}$ have yielded detections rather than limits.

Similarly, the limits on $\beta_{\text {opt }-\gamma}$ for nondetections $(>-0.7)$ are in the range of most of the $\beta_{\mathrm{opt}-\gamma}$ from the prompt detections (from -0.9 to 0.03 ). The prompt nondetections are consistent with coming from the harder end of the $\beta_{\text {opt }-\gamma}$ distribution, as all of the $\beta_{\text {opt }-\gamma}$ limits are harder than the softest $\beta_{\text {opt }-\gamma}$ value calculated from prompt detections. However, there is no evidence of bi-

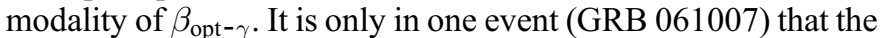
$\beta_{\text {opt }-\gamma}$ of optical detections is softer than the softest allowed $\beta_{\mathrm{opt}-\gamma}$ from a prompt limit. This was for epochs at the end of the event, which may be the beginning of the afterglow, as $\gamma$-ray, X-ray, and optical frequencies lie on a single spectral power law. There is not one set of $\beta_{\mathrm{opt}-\gamma}$ for the optically detected and another for the nondetected cases. These overlaps in $\beta_{\mathrm{opt}-\gamma}$ and $\beta_{\gamma}$ are readily seen in Figure 2.

\subsection{Cases with Prompt X-ray Data}

For GRB 061121, the comparisons of $\beta_{\text {opt- } \gamma}$ and $\beta_{\text {opt-X }}$ require a peak in the broadband $f_{\nu}$ spectrum. This is discussed in detail by Page et al. (2007), where it can be inferred to be near $1 \mathrm{keV}$ initially and to subsequently drop in frequency. GRB 060729 also implies a peak between the optical and X-ray during the epoch with optical, X-ray, and $\gamma$-ray data. In that case, $\beta_{\gamma}$ appears to be harder than $\beta_{\mathrm{X}}$, but this may be due to the general softening trend of $\beta_{\gamma}$ and the measurement of $\beta_{\gamma}$ over the X-ray epoch using data beginning well before the X-ray observations. A "convex" overall X-ray- $\gamma$-ray spectral shape cannot be inferred from the weak $\gamma$-ray detection.

In contrast, the GRB 061007 prompt X-ray epochs do not demonstrate such a peak. From the first GRB 061007 epoch with X-ray data, the spectral indices show that the $\gamma$-ray and X-ray bands are in a single spectral segment. This is not unusual; $\S 3$ indicates that in the two prompt optical limit cases, the X-ray and $\gamma$-ray data could be from the same spectral segment. In GRB 061007, allowing for local extinction corrections, the entire broadband spectrum (optical, X-ray, $\gamma$-ray) forms a single spectral segment (see Mundell et al. 2007, Fig. 2, which fits an absorbed $\nu^{-1}$ spectrum). This would be expected for an early afterglow where the high-energy emission from the forward shock extends above the X-rays.

\section{CONCLUSION}

Prompt optical limits fall within the range of optical fluxes and optical-to- $\gamma$-ray flux ratios observed from prompt optical detections. The prompt limits yield constraints on optical-to- $-\gamma$-ray flux ratios at the faint end of the ratios measured from prompt detections. This does not imply a different set of intrinsic or environmental properties for events with detections and nondetections; there is wide overlap in fluxes and flux ratios between the limits and detections. Moreover, prompt detections show great variety, and demonstrate diverse connections (or lack thereof) with the contemporaneous $\gamma$-rays.

The most economical explanation for prompt optical nondetections is that they are events drawn from the faint end of the range of prompt optical emission. These faint counterparts are not always accessible with the sensitivities of the small telescopes providing the bulk of prompt responses.

This work has been supported by NASA grants NNG-04WC41G and F006794, NSF grants AST-0119685, 0105221, and 0407061, the Australian Research Council, the University of New South Wales, and the University of Michigan. J. C. W. is supported by NSF grant AST-0406740. Work performed at LANL is supported through internal LDRD funding.
Akerlof, C. W., et al. 2003, PASP, 115, 132

Barthelmy, S., et al. 2006, GCN Circ., 5256, 1

Bertin, E., \& Arnouts, S. 1996, A\&AS, 117, 393

Bessell, M. S. 1979, PASP, 91, 589

Butler, N. R., Kocevski, D., Bloom, J. S., \& Curtis, J. L. preprint (arXiv:0706.1275)

Campana, S., et al. 2006, Nature, 442, 1008

Chen, Y. C., Lee, Y. H., Huang, K. Y., Ip, W. H., \& Urata, Y. 2006, GCN Circ., 5797,1

Fishman, G. J., \& Meegan, C. A. 1995, ARA\&A, 33, 415

Fynbo, J. P. U., Jakobsson, P., Jensen, B. L., Hjorth, J., Sollerman, J., Watson, D., Cer $\tilde{A}^{3}$ n, J. M. C., Vreeswijk, P., \& Andersen, M. I. 2006, GCN Circ., 5651,1

\section{REFERENCES}

Gehrels, N., et al. 2004, ApJ, 611, 1005

Kumar, P., et al. 2007, MNRAS, 376, L57

Malesani, D., D’Avanzo, P., Palazzi, E., Israel, G. L., Chincarini, G., Stella, L., \& Pedani, M. 2005, GCN Circ., 3582, 1

Mangano, V., Parola, V. L., Troja, E., Cusumano, G., Mineo, T., Parsons, A., \& Kennea, J. 2006, GCN Circ., 5254, 1

Medvedev, M. V. 2006, ApJ, 637, 869

Meszaros, P. 2006, Rep. Prog. Phys., 69, 2259

Meszaros, P., Rees, M. J., \& Papathanassiou, H. 1994, ApJ, 432, 181

Mundell, C. G., et al. 2007, ApJ, 660, 489

Nousek, J. A., et al. 2006, ApJ, 642, 389

Nysewander, M., \& Haislip, J. 2006, GCN Circ., 5236, 1 
O’Brien, P. T., et al. 2006, ApJ, 647, 1213

Page, K. L., et al. 2007, ApJ, 663, 1125

Parsons, A. M., et al. 2006, GCN Circ., 5252, 1

Pe'er, A., \& Waxman, E. 2004, ApJ, 613, 448

Perley, D. A., et al. 2007, ApJ, submitted (astro-ph/0703538)

Piran, T. 2005, Rev. Mod. Phys., 76, 1143

Price, P. A., Berger, E., \& Fox, D. B. 2006, GCN Circ., 5275, 1

Quimby, R., \& Rykoff, E. S. 2006, GCN Circ., 5377, 1

Quimby, R., Schaefer, B. E., \& Swan, H. 2006a, GCN Circ., 4782, 1

Quimby, R., Swan, H., Rujopakarn, W., \& Smith, D. A. 2006b, GCN Circ., 5366, 1

Quimby, R. M., et al. 2006c, ApJ, 640, 402

Ruiz-Velasco, A. E., et al. 2007, ApJ, 669, 1

Rykoff, E. S., \& Rujopakarn, W. 2006, GCN Circ., 5706, 1

Rykoff, E. S., Rujopakarn, W., \& Yuan, F. 2006, GCN Circ., 5504, 1

Rykoff, E. S., et al. 2004, ApJ, 601, 1013
Rykoff, E. S., et al. 2005, ApJ, 631, L121

Schaefer, B. E., Rykoff, E. S., Smith, D. A., \& Quimby, R. 2006, GCN Circ., 5222,1

Schaefer, B. E., \& Xiao, L. 2006, ApJL, submitted (astro-ph/0608441)

Schlegel, D. J., Finkbeiner, D. P., \& Davis, M. 1998, ApJ, 500, 525

Stetson, P. B. 1987, PASP, 99, 191

Thoene, C. C., Levan, A., Jakobsson, P., Rol, E., Gorosabel, J., Jensen, B. L., Hjorth, J., \& Vreeswijk, P. 2006, GCN Circ., 5373, 1

Thompson, C. 1994, MNRAS, 270, 480

Usov, V. V. 1994, MNRAS, 267, 1035

Vestrand, W. T., et al. 2005, Nature, 435, 178 2006, Nature, 442, 172

Wei, D. M. 2007, MNRAS, 374, 525

Yost, S. A., Yuan, F., Swan, H., \& Akerlof, C. 2006, GCN Circ., 4488, 1

Yost, S. A., et al. 2007, ApJ, 657, 925

Zhang, B. Feb. 2007, Chinese J. Astron. Astrophys., 7, 1 